05,01

\title{
Задержанные скачки размагничивания в магнитах (NdDy)(FeCo)B в стационарном магнитном поле
}

\author{
() Г.Л. Львова ${ }^{1}$, М.В. Кирман ${ }^{1}$, О.В. Коплак ${ }^{1}$, В.В. Кучеряев ${ }^{2}$, Р.А. Валеев ${ }^{2}$, \\ В.П. Пискорский ${ }^{2}$, Р.Б. Моргунов ${ }^{1,2,3, q}$ \\ ${ }^{1}$ Институт проблем химической фризики РАН, \\ Черноголовка, Россия \\ ${ }^{2}$ Всероссийский институт авиационных материалов, \\ Москва, Россия \\ ${ }^{3}$ Тамбовский государственный технический университет, \\ Тамбов, Россия \\ ฯ E-mail: morgunov2005@yandex.ru \\ (Поступила в Редакцию 31 января 2017 г. \\ В окончательной редакции 27 января 2017 г.)
}

Обнаружены спонтанные скачки размагничивания, которые наблюдаются в постоянном магнитном поле в спеченных магнитах $\left(\mathrm{Nd}_{0.6} \mathrm{Dy}_{0.4}\right)_{16}\left(\mathrm{Fe}_{0.77} \mathrm{Co}_{0.23}\right)_{78} \mathrm{~B}_{6}$ после резкого уменьшения внешнего магнитного поля от значения, соответствующего насыщению к значению, близкому к коэрцитивной силе. Показано, что число скачков намагниченности обратно пропорционально их амплитуде. Малое значение коэффициента автокорреляции между амплитудой скачка и временем его появления $(R<0.1)$ свидетельствует о стохастичности скачков. Установлено, что спектральная плотность скачков не зависит от частоты, т.е. наблюдается белый магнитный шум. Получено распределение градиента магнитного поля вблизи поверхности образца, которое позволяет различать домены и намагниченность зерен в зависимости от направления текстурирования спеченного магнита.

Работа выполнена при поддержке гранта 3.1992.2017/ПЧ в рамках конкурса научных проектов, выполняемых научными коллективами исследовательских центров и (или) научных лабораторий образовательных организаций высшего образования.

DOI: 10.21883/FTT.2017.11.45071.025

\section{1. Введение}

Постоянные магниты семейства $R E-T M-\mathrm{B}$ были открыты в 80-х годах XX века $[1,2](R E-$ редкоземельные металлы, $T M$ - переходные металлы, В - бор). На сегодняшний день такие магниты представляют собой усовершенствование исходной версии $\mathrm{NdFeB} \mathrm{[3]} \mathrm{и} \mathrm{по}$ многим характеристикам (энергетическое произведение, остаточная намагниченность, временна́я стабильность и др.) остаются непревзойденными. Несмотря на большое число публикаций, посвященных исследованию сплавов $R E-T M-\mathrm{B}$ (см., например, обзоры [4-6]), механизмы перемагничивания этих сплавов до сих пор остаются практически неизученными, хотя именно эти процессы определяют пригодность магнитов для практических применений. Общепризнанным фактом является существование в сплавах зародышей фазы обратной намагниченности, рост которой приводит к уменьшению вклада основной фазы. Наряду с этим имеет место движение доменных стенок, однако об их природе и свойствах известно мало. Упомянутые механизмы перемагничивания конкурируют друг с другом, создавая необычные условия для нелинейного отклика намагниченности на приложенное внешнее магнитное поле. Одним из методов изучения динамики размагничивания материалов является измерение и анализ временных $(t)$ зависимостей магнитного момента $M(t)$ в нулевом или отличном от нуля магнитном поле, направленном против исходной намагниченности. Для большинства магнитотвердых материалов наблюдается логарифмическая зависимость $M(t)[7,8]$, которая объясняется широким распределением высот потенциальных барьеров и локальных барьеров движения доменных стенок.

В [9] сообщалось об обнаружении скачков намагниченности в спеченных магнитах $(\mathrm{NdDySm})(\mathrm{FeCo})$ при низких температурах $\sim 2 \mathrm{~K}$ на нисходящей кривой магнитного гистерезиса (при отрицательной развертке магнитного поля). В [10-12] были обнаружены скачки намагниченности в сплавах SmCo при температурах $\sim 10 \mathrm{~K}$. Эти и другие работы о скачках перемагничивания были выполнены в условиях изменяющегося внешнего магнитного поля.

В данной статье сообщается о наблюдении иного эффекта, который заключается в скачкообразном спонтанном размагничивании магнита в стационарном магнитном поле, установившемся после его переключения от насыщающего магнитного поля к меньшему отрицательному значению. Отсутствие развертки магнитного поля в наших опытах устраняет запаздывание системы доменных стенок по отношению к достигнутому уровню магнитного поля и невозможным возникновение $S$-образной нелинейности, которая является главным 


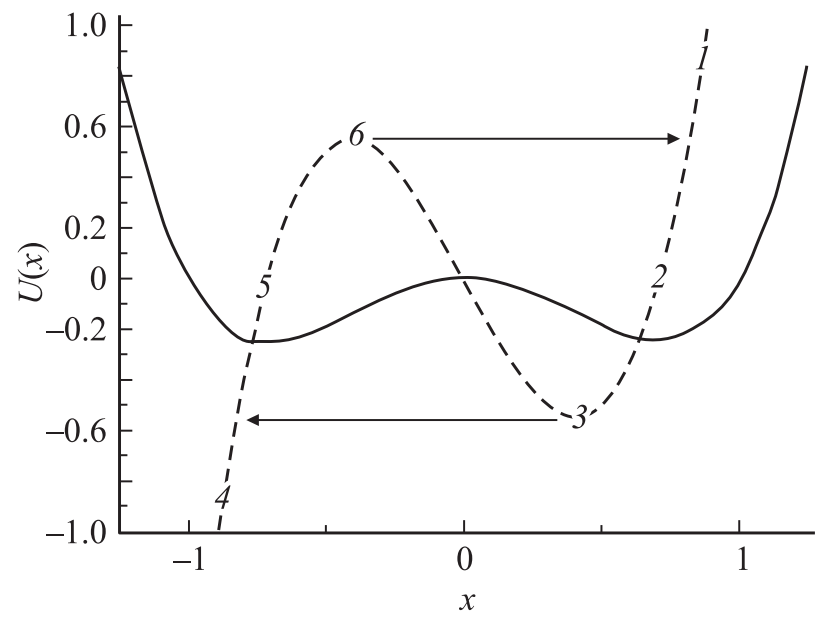

Рис. 1. Схематическое изображение зависимости энергии доменной стенки $U(x)$ и ее производной по координате $U^{\prime}(x)$ (т. е. силы) от конфигурационной координаты $x$ в изменяющемся магнитном поле [13]. Горизонтальными стрелками показан скачок Баркгаузена. В точке 1 стартует развертка магнитного поля в направлении увеличения его положительного значения, точке 2 соответствует наличие двух минимумов на потенциальной кривой, точке 3 соответствует скачок намагниченности, в точке 4 поле продолжает нарастать, сдвигая позицию одиночного минимума энергии, в точках 5 и 6 развертка поля происходит в обратную сторону.

условием скачков Баркгаузена в изменяющемся магнитном поле [13] (рис. 1). Последовательное продвижение по петле гистерезиса в работах [10-12] и схематический потенциальный рельеф доменной стенки $U(x)$ показаны на рис. 1, где показано, каким образом возникает скачок перемагничивания при нарастании и убывании магнитного поля.

В условиях наших экспериментов переключение внешнего магнитного поля создает неравновесную доменную структуру, которая впоследствии релаксирует, приводя к скачкам Баркгаузена в постоянном магнитном поле. Преимущества такой постановки экспериментов заключаются в том, что скачки размагничивания не зависят от внешних экспериментальных условий и зависят от метастабильного состояния магнита, созданного переключением магнитного поля. В качестве объекта исследования были выбраны классические редкоземельные магниты, для которых нами ранее были получены подробные количественные данные о магнитной вязкости и магнитной кристаллической анизотропии $[14,15]$, о влиянии самария на магнитную релаксацию [16], а также предварительные данные о возможном спектре белого магнитного шума, извлеченные из анализа магнитной вязкости [17]. В работах [14-17] было показано, что доминирующим механизмом торможения доменов в исследуемых образцах при комнатной температуре является их закрепление на случайных препятствиях (pinning), а при низких температурах ниже $100 \mathrm{~K}$ доминирует зарождение фазы обратной намагниченности.
Цели работы заключались в создании экспериментальных условий для наблюдения самопроизвольных скачков намагниченности в стационарном магнитном поле, а также в статистическом анализе спектра магнитного шума, параметров корреляции, регулярности временны́х рядов в магнитных сплавах $\mathrm{NdDy}(\mathrm{FeCo}) \mathrm{B}$. Кроме того, одной из целей была визуализация объектов, эволюция которых в стационарном магнитном поле может соответствовать скачкам намагниченности.

\section{2. Экспериментальная часть}

Образцы $\left(\mathrm{Nd}_{0.6} \mathrm{Dy}_{0.4}\right)_{16}\left(\mathrm{Fe}_{0.77} \mathrm{Co}_{0.23}\right)_{78} \mathrm{~B}_{6}$ получали путем спекания частиц размером 500-600 $\mu \mathrm{m}$ в высокотемпературной вакуумной печи. Методика получения, а также химический и фазовый анализ подробно описаны в работе [14]. Параметры тетрагональной ячейки основной магнитной фазы близки к параметрам сплава $\mathrm{Nd}_{2} \mathrm{Fe}_{14} \mathrm{~B}$ $(2-14-1)$, для которого $a=8.80 \AA, c=12.20 \AA[2,3]$.

Для образцов, имеющих форму пластинки размером $0.5 \times 1.5 \times 3.5 \mathrm{~mm}$, были выполнены измерения магнитного момента в интервале магнитных полей $-50-+50 \mathrm{kOe}$ (рис. 2) и временны́е зависимости магнитного момента с помощью магнитометра MPMS 5XL Quantum Design. Измерения проводились при температуре $T=300 \mathrm{~K}$, в процессе измерения температура образца поддерживалась с точностью $0.1 \mathrm{~K}$. Внешнее магнитное поле было направлено вдоль легкой оси намагничивания образца. Перед измерением временны́х зависимостей магнитного момента образец был намагничен до насыщения в магнитном поле насыщения $H_{\text {sat }}=50 \mathrm{kOe} \mathrm{в} \mathrm{течение} 5 \mathrm{~min}$. Затем в постоянном магнитном поле $H=-10 \mathrm{kOе}$ проводились измерения $M(t)$. Для получения статистического набора скачков размагничивания накапливались 18 зависимостей $M(t)$, полученных после переключения постоянного магнитного поля к значению $H=-9 \mathrm{kOe}$. Остальные условия за-

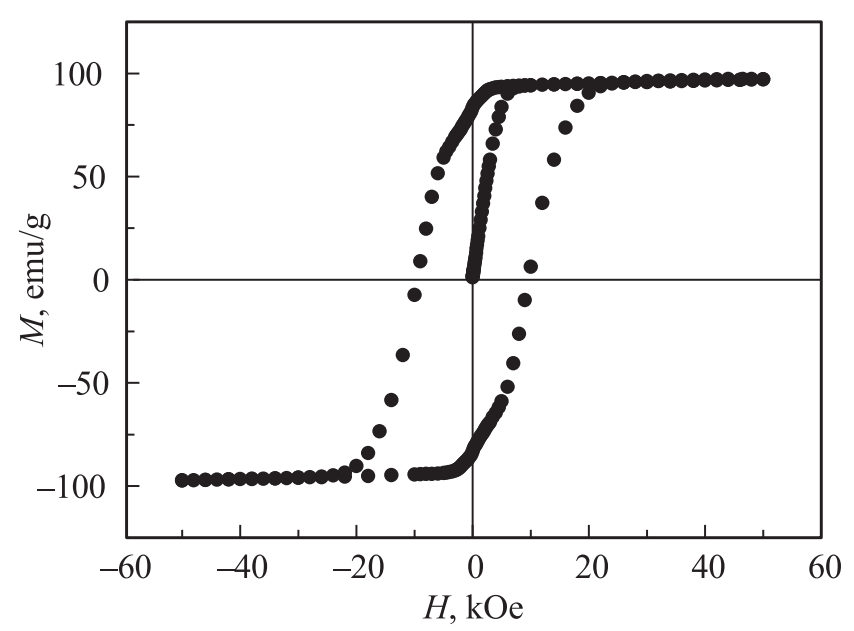

Рис. 2. Петля магнитного гистерезиса образца при температуре $300 \mathrm{~K}$. 

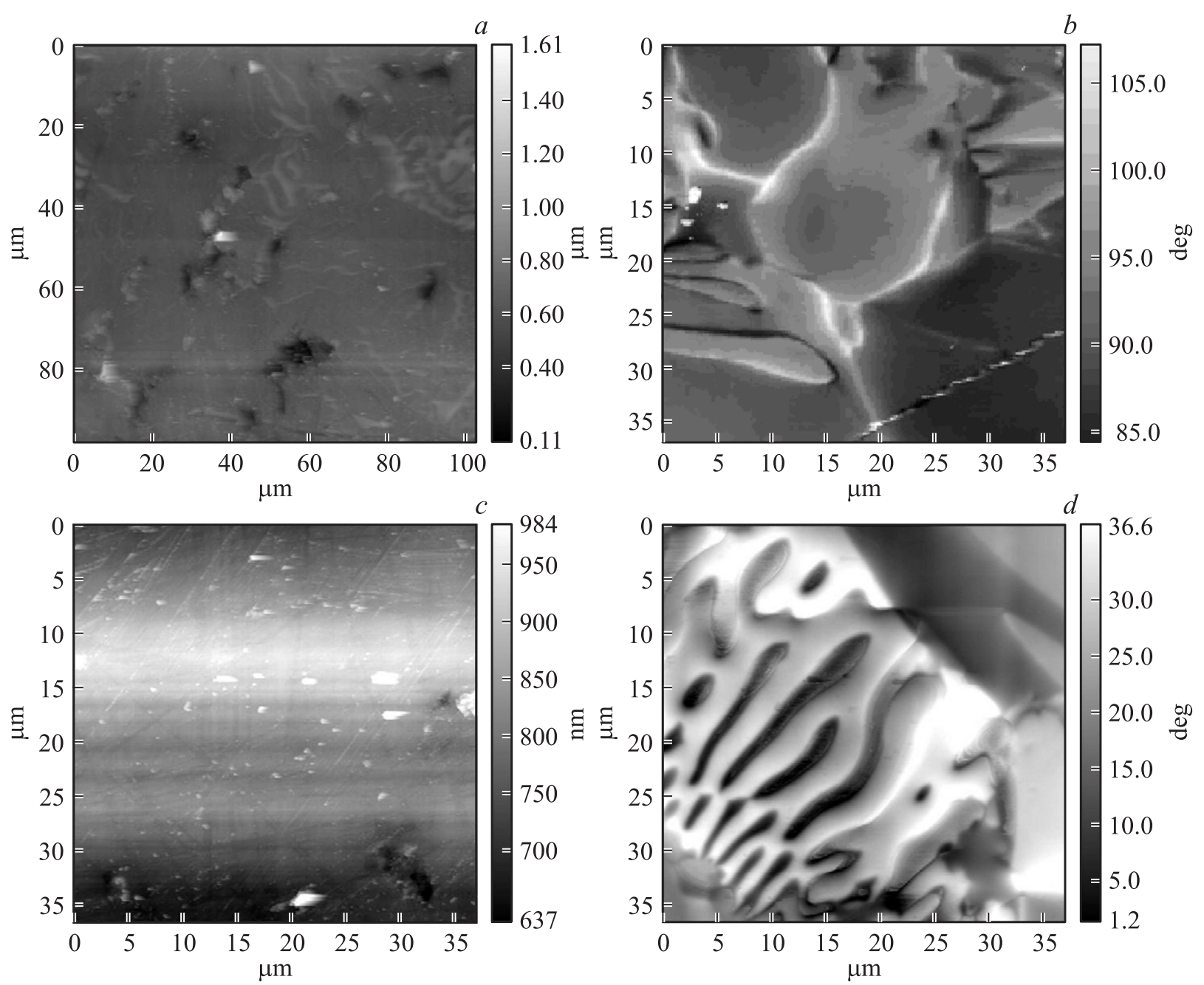

Рис. 3. Рельеф поверхности образца в режиме $\mathrm{ACM}(a, c)$ и распределение градиента магнитного поля в режиме МСM $(b, d)$ для ориентаций изучаемой поверхности одного и того же образца перпендикулярно $(a, b)$ и параллельно $(c, d)$ магнитной текстуре. Полутоновая шкала отвечает фазе колебаний кантилевера, измеряемой в градусах.

писи зависимостей $M(t)$ были одинаковы, а сами они получены на одном и том же образце. В результате был получен статистически значимый набор событий, из которого определяли параметры скачков (средний магнитный момент начала скачка, его средняя амплитуда, время ожидания и др.). Затем были получены статистические распределения по этим параметрам или временные ряды параметров. Полученные временные ряды анализировали как магнитный шум, спектральная плотность которого характеризует распределение мощности скачков намагниченности по частотному спектру.

Изображения доменной структуры в предварительно намагниченных образцах были получены с помощью атомного силового микроскопа Aist-NTSmartSPM в отсутствие магнитного поля. Режим атомной силовой микроскопии (ACM) был использован для установления рельефа поверхности, и того факта, что магнитный рельеф не повторяет геометрию распределения высот. В режиме магнитной силовой микроскопии (МСM) из- меряемые значения магнитной силы, пропорциональные градиенту магнитного поля вблизи поверхности образца, были также пропорциональны частотному сдвигу вибрирующего кантилевера на основе $\mathrm{CoCr}$ с коэрцитивной силой $400 \mathrm{Oe}$ в режиме tapping-lift. Силовая константа и резонансная частота кантилевера были равны $5 \mathrm{H} / \mathrm{m}$ и $196 \mathrm{kHz}$ соответственно. Расстояние кантилевера от поверхности составляло $80 \mathrm{~nm}$, и устанавливалось одинаковым во всех опытах.

\section{3. Результаты и обсуждение}

На рис. 2 показана петля магнитного гистерезиса, записанная при ориентации магнитного поля вдоль оси легкого намагничивания. Коэрцитивная сила составляла $H_{\mathrm{C}}=9.5 \mathrm{kOe}$, намагниченность насыщения $M_{\mathrm{SAT}}=97 \mathrm{emu} / \mathrm{g}$ при $300 \mathrm{~K}$. Подобные петли магнитного гистерезиса были записаны при различных скоростях прохождения петли магнитного гистерезиса в диапазоне 
$1.1 \cdot 10^{2}-3 \cdot 10^{5} \mathrm{Oe} / \mathrm{min}$, однако, как и на рис. 2 , скачки намагниченности (эффект Баркгаузена) не наблюдались. После отключения насыщающего магнитного поля в образце формировалась доменная структура, которая была исследована с помощью сканирования градиента магнитного поля вблизи поверхности образца в режиме (МСM). Кроме того, было выполнено сканирование поверхности в режиме АСM (рис. 3). При этом из одного и того же образца спеченного магнита было вырезано две пластинки вдоль и поперек магнитной текстуры, которая создавалась в процессе прессования образца в магнитном поле. Это было необходимо потому, что образец, помещаемый в силовой микроскоп, должен был иметь форму тонкой пластинки с шлифованной поверхностью. Необходимость в исследовании двух образцов была вызвана тем, что боковые поверхности тонкой пластинки не могли быть исследованы в микроскопе. Магнитное текстурирование (выстраивание легких осей намагниченности зерен в одном направлении) приводит к анизотропии в распределении доменов и анизотропии размеров зерен. На рис. 3, $a$ и $b$ представлены АСМи МСМ-сканы поверхности одного и того же образца, выполненные перпендикулярно направлению текстурирования, а также в плоскости, параллельной направлению магнитной текстуры, соответственно. Рельефы ACM и МCM различны, что говорит о том, что магнитное изображение вызвано не шероховатостью образца, а отражает распределение намагниченности элементов образца. Намагниченные области для этих ориентаций различаются. Удлиненные полосовые домены (stripedomain) с разветвленной структурой наблюдаются для поверхности, сканированной в плоскости текстурирования (рис. $3, b$ ). Отдельные не сливающиеся домены имеют место при сканировании поверхности перпендикулярно направлению текстурирования (рис. 3,a). Аналогичные домены были обнаружены в образцах семейства $R E-T M-\mathrm{B}$ в [18]. В нашей работе не было возможности для измерения изменения распределения намагниченности in situ в магнитном поле, однако статичные изображения, как будет показано далее, могут быть использованы для оценки объема перемагничиваемых областей при скачкообразном размагничивании. На фото видны полосовые домены длиной $\sim 15-20 \mu \mathrm{m}$ и шириной $\sim 3-5 \mu \mathrm{m}$. Домены расположены внутри зерен, которые отчетливо видны в виде областей средним размером $\sim 18 \times 18 \mu \mathrm{m}$.

При измерении временнб́х зависимостей $M(t)$ было обнаружено, что на фоне плавной релаксации магнитного момента происходят резкие скачки магнитного момента (рис. 4). Из серии зависимостей $M(t)$ было получено распределение вероятности скачков $P$ по времени их появления (рис. 5), из которой видно, что чаще всего скачки происходят на начальной стадии релаксации сразу после переключения магнитного поля, а со временем эмиссия скачков размагничивания затухает.

На рис. 6 представлено распределение вероятности скачков $P$ по амплитуде $\langle\Delta M\rangle$ и магнитному момен-

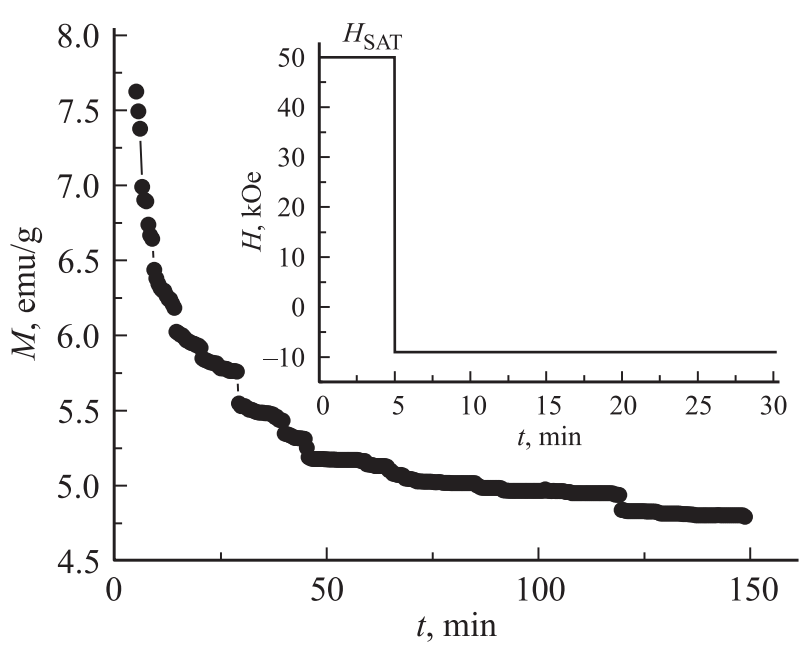

Рис. 4. $a$ - схема переключения внешнего магнитного поля в экспериментах по измерению временнб́х зависимостей намагниченности $M(t)$. Перед измерением образец был намагничен до насыщения в магнитном поле $H_{\text {sat }}=50 \mathrm{kOe}$ в течение $5 \mathrm{~min}$. $b$ - зависимость магнитного момента от времени $M(t)$ после включения постоянного поля $H=-9 \mathrm{kOe}$ при $T=300 \mathrm{~K}$.

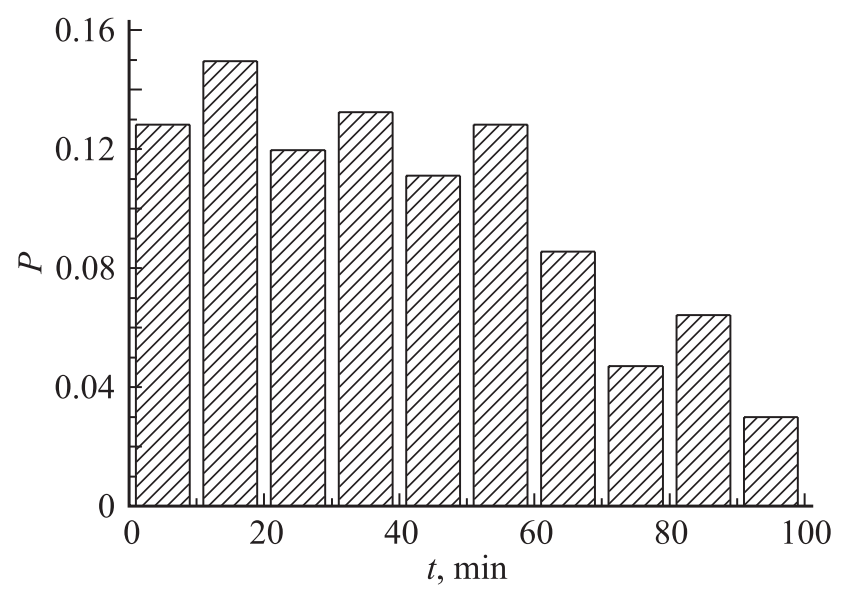

Рис. 5. Распределение вероятности скачков размагничивания $P$ по времени $t$, прошедшему от стабилизации магнитного поля до начала скачка.

ту $M_{\text {start }}$, достигнутому в процессе магнитной релаксации к моменту времени, когда происходит скачок. Максимум числа скачков приходится на скачки амплитуды $\langle\Delta M\rangle \sim 0.05 \mathrm{emu} / \mathrm{g}$, которые происходят в узком интервале значений магнитного момента $M_{\text {start }} \sim 5-7 \mathrm{emu} / \mathrm{g}$.

В $[15,16]$ было показано, что магнитный гистерезис в спеченных постоянных магнитах типа $(\mathrm{NdDy})(\mathrm{FeCo}) \mathrm{B}$, легированных $\mathrm{Sm}$, обусловлен двумя механизмами: зародышеобразование обратной магнитной фазы вблизи дефектов кристалла и смещением доменных границ, задерживаемых препятствиями. Причем, при температурах $\sim 300 \mathrm{~K}$ доминирует механизм перемагничивания путем движения доменных стенок. Поэтому дальнейшее обсуждение будет проведено в рамках этого механизма. 


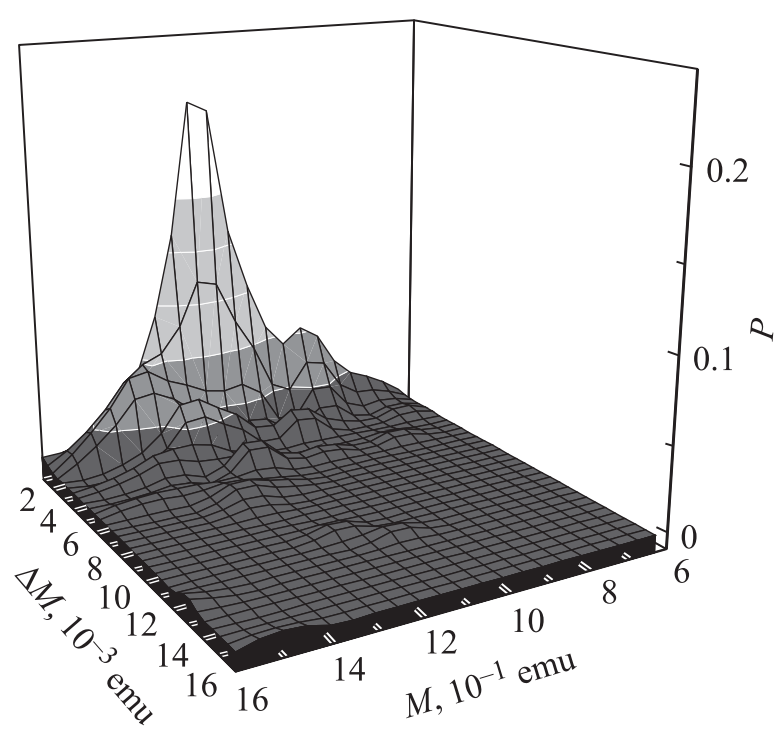

Рис. 6. Двумерное распределение вероятности скачков размагничивания $P$ по их амплитуде $\Delta M$ и магнитному моменту $M$, достигнутому в процессе релаксации в постоянном магнитном поле на момент начала скачка.

Объем образца $\Delta V$, вовлеченный в скачок намагниченности можно оценить из соотношения

$$
\Delta V=\frac{\langle\Delta M\rangle \cdot V}{M}
$$

Эта оценка дает $\Delta V \sim 6.8 \cdot 10^{-4} \mathrm{~mm}^{3}$, т. е. в формирование скачка вовлечено $0.026 \%$ от объема образца. Толщина домена была оценена из рис. $3, b$, на котором показано распределение градиента магнитного поля в режиме МСМ для перпендикулярной ориентации изучаемой поверхности. Длина и ширина домена определялись по 10 рисункам, аналогичным рис. $3, d$, и далее вычислялось среднее значение этих параметров. Отношение размера домена $(15 \times 5 \times 2.5 \mu \mathrm{m}$, см. рис. 3$)$ к размеру образца $\left(V=2.63 \mathrm{~mm}^{3}\right)$ составляет $\sim 2 \cdot 10^{-7}$, что значительно меньше, чем доля объема, вовлеченного в одиночный скачок $\sim 2.6 \cdot 10^{-4}$. Из этого можно сделать вывод, что скачок намагниченности соответствует перемагничиванию $\sim 10^{3}$ доменов.

С другой стороны, энергия $\Delta E$, необходимая для перемагничивания объема $\Delta V$ образца в приложенном магнитном поле $H=9 \mathrm{kOe}$ составляет $\Delta E=2\langle\Delta M\rangle \cdot H$ $=0.002 \mathrm{emu} \cdot 9000 \mathrm{Oe}=18 \mathrm{erg}$. Как известно, ширина доменных стенок в магнитах семейства $R E-T M-\mathrm{B}$ составляет 50-80 nm, что значительно больше, чем период решетки $12 \AA$ в направлении легкой оси намагничивания [2,3]. Поэтому механизмы торможения доменных стенок, связанные с влиянием периодического потенциального рельефа Пайерлса на их подвижность полностью исключаются. Известно, что в редкоземельных магнитах движение доменных стенок лимитируется их закреплением на неоднородностях кристаллической решетки, связанных с наличием дефектов структуры.
Процесс преодоления таких хаотичных препятствий доменной стенкой является термоактивированным и характеризуется средней высотой потенциального барьера. В классических работах $[19,21]$ приведено подробное теоретическое описание процесса преодоления препятствий различной формы доменной стенкой. Там же показано, что одним из параметров движения доменной стенки является работа, которая совершается при преодолении доменной стенки, которая равна произведению эффективной силы $f$, действующей на доменную стенку в магнитном поле, на активационный объем $b$. Эта работа соответствует минимально затраченной энергии, требуемой для открепления доменной стенки от препятствия (domain walls depinning [19-20]). Считая, что эта энергия тратится на открепление доменных стенок от препятствий, а также принимая во внимание, что энергия для единичного акта открепления составляет $f b=3 \cdot 10^{-11} \mathrm{erg}[12,13]$, получим число элементарных процессов открепления доменов от препятствий в одном скачке: $N_{D}=\Delta E / f b=18 \mathrm{erg} / 3 \cdot 10^{-11} \mathrm{erg}=6 \cdot 10^{11}$.

Возможным объяснением наблюдаемых скачков перемагничивания является пребывание доменных стенок в метастабильном состоянии сразу после переключения внешнего магнитного поля. В этом случае, согласно [22], сила, действующая на доменные стенки со стороны постоянного внешнего магнитного поля, способна привести к скачкообразному термоактивированному преодолению препятствий доменными стенками.

Количество скачков, получаемых в одном эксперименте $\sim 10$ было недостаточно для статистического анализа закономерностей их появления. Поэтому один и тот же эксперимент был произведен 18 раз с целью набора представительной статистики изучаемых событий. Так как измерения в отдельных экспериментах были независимы друг от друга, и мы предполагали, что после насыщения намагниченности полем $5 \mathrm{~T}$ образец „не запоминал“ предыдущие эксперименты, было логично думать, что справедлива эргодическая гипотеза (среднее по времени равно среднему по ансамблю). В рамках этого предположения для статистического анализа скачков размагничивания было использовано слияние данных от нескольких независимых измерений, т.е. был построен общий временной ряд амплитуды скачков $\left(t_{n}, \Delta M_{n}\right)$, где $n=1 \ldots N$ - номера интервала времени $t_{n}=n \cdot \Delta t$, $\Delta M_{n}$ - амплитуда скачка, происходящего в интервале $\left(t_{n}-\Delta t / 2 ; t_{n}+\Delta t / 2\right)$. Значение коэффициента автокорреляции между амплитудой скачка и временем его появления, рассчитанное по стандартным методикам [23,24], оказалось малым $R<0.1$, что свидетельствует о стохастическом характере скачков.

Для получения спектральной плотности скачков $S$ сначала была получена коррелограмма $r(k)$ временно́го ряда $\left(t_{n}, \Delta M_{n}\right)$

$$
r(k)=r\left(\Delta M_{n}, \Delta M_{n-k}\right)=\frac{\overline{\Delta M_{n} \cdot \Delta M_{n-k}}-\overline{\Delta M_{n}} \cdot \overline{\Delta M_{n-k}}}{\sigma\left(\Delta M_{n}\right) \cdot \sigma\left(\Delta M_{n-k}\right)},
$$




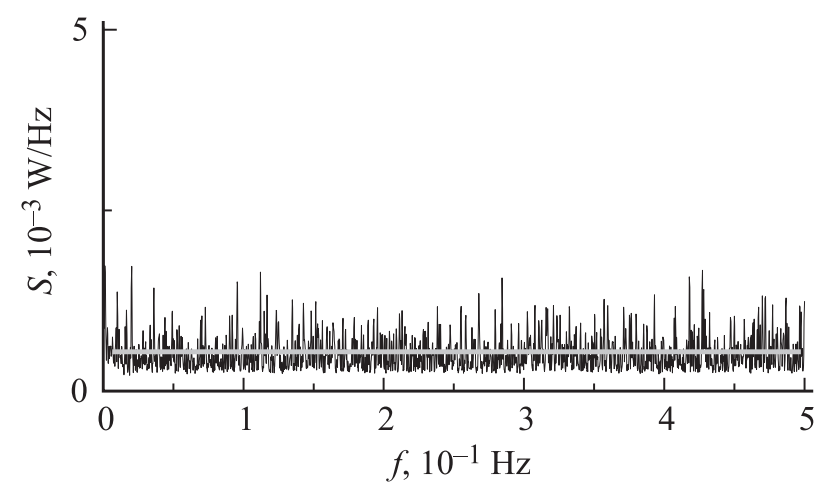

Рис. 7. Спектральная плотность $S$ скачков намагниченности в постоянном магнитном поле. Сплошной линией показана аппроксимация функцией (6) (см. текст).

где среднеквадратичные отклонения амплитуды скачка

$$
\begin{aligned}
\sigma\left(\Delta M_{n}\right) & =\sqrt{\frac{1}{N-k} \sum_{n=1+k}^{N} \Delta M_{n}^{2}-\left(\sum_{n=1+k}^{N} \Delta M_{n}\right)^{2},} \\
\sigma\left(\Delta M_{n-k}\right) & =\sqrt{\frac{1}{N-k} \sum_{n=1+k}^{N} \Delta M_{n-k}^{2}-\left(\sum_{n=1+k}^{N} \Delta M_{n-k}\right)^{2}},
\end{aligned}
$$

и средние значения амплитуды скачка

$$
\begin{aligned}
& \overline{\Delta M_{n}}=\sum_{n=1+k}^{N} \Delta M_{n}, \quad \overline{\Delta M_{n-k}}=\sum_{n=1}^{N-k} \Delta M_{n}, \\
& \overline{\Delta M_{n} \cdot \Delta M_{n-k}}=\frac{1}{N-k} \sum_{n=1+k}^{N} \Delta M_{n} \cdot \Delta M_{n-k} .
\end{aligned}
$$

Затем для получения частотной зависимости спектральной плотности скачков $S(f)$ (рис. 7) было применено преобразование Фурье коррелограммы временно́го ряда $r\left(\Delta M_{n} \Delta M_{n-k}\right)$ в соответствии с теоремой Винера-Хинчина $[23,24]$

$$
S\left(f_{n}\right)=\sum_{k=1}^{N} r(k) \cdot \exp \left(-(2 \pi i / N) k f_{n} \Delta t\right),
$$

где $f_{n}=n /(N \Delta t), \quad n=1 \ldots N$. соответствуют набору частот дискретного преобразования Фурье, шаг дискретизации которого определяется временем записи $T=N \cdot \Delta t$, а диапазон частот - шагом дискретизации $\Delta t$ временно́го ряда. В результате аппроксимации спектральной плотности функцией $S=(1 / f)^{\alpha}$ (рис. 7), было получено значение $\alpha=0$, которое характерно для случайных процессов, представляющих „белый“ шум [24].

\section{4. Выводы}

В образцах $\left(\mathrm{Nd}_{0.6} \mathrm{Dy}_{0.4}\right){ }_{16}\left(\mathrm{Fe}_{0.77} \mathrm{Co}_{0.23}\right){ }_{78} \mathrm{~B}_{6}$ обнаружены стохастические скачки размагничивания в необычных условиях, когда внешнее магнитное поле было постоянным. В стандартных условиях в процессе записи петли гистерезиса, скачков намагниченности не наблюдалось в диапазоне скоростей развертки, охватывающем 3 порядка величины при той же чувствительности магнетометpa, что и в постоянном магнитном поле. Число скачков обратно пропорционально их амплитуде, а максимальная вероятность их возникновения отвечает магнитному моменту образца на уровне $10 \%$ от намагниченности насыщения. Частотная зависимость спектральной плотности скачков размагничивания соответствует белому шуму. Средняя амплитуда скачков размагничивания объясняется перемагничиванием $\sim 10^{3}$ доменов.

\section{Список литературы}

[1] M. Sagawa, S. Fujimura, N. Togawa, H. Yamamoto, Y. Matsuura. J. Appl. Phys. A 55, 2083 (1984).

[2] J.F. Herbst. Rev. Mod. Phys. 63, 819 (1991).

[3] E. Burzo. Rep. Prog. Phys. 61, 1099 (1998).

[4] V.Yu. Irkhin. J. Magn. Magn. Mater. 258-259, 228 (2003).

[5] Q. Yao, W. Liu, W. B. Cui, F. Yang, X.G. Zhao, Z.D. Zhang. J. Phys. Phys. 42, 035007 (2009).

[6] J. Fidler, T. Schrefl. J. Appl. Phys. 79, 5029 (1996).

[7] D.K. Lottis, E.D. Dahlberg, J.A. Christner, J.I. Lee, R.L. Peterson, R.M. White. J. Appl. Phys. 63, 2920 (1988).

[8] D. Givord, Q. Lu, M.F. Rossignol, P. Tenaud, T. Viadieu. J. Magn. Magn. Mater. 83, 183 (1990).

[9] D.S. Neznakhin, A.S. Bolyachkin, A.S. Volegov, P.E. Markin, S.V. Andreev, N.V. Kudrevatykh. JMMM J. Magn. Magn. Mater. 377, 477 (2015).

[10] A. Handstein, D. Eckert, K.-H. Muller, B. Wall, W. Rodewald. IEEE Trans. Magn. 30, 598 (1994).

[11] Y. Nakagawa, H. Hiroyoshi, M. Sagawa, S. Hirosawa. IEEE Trans. Magn. (1987). V. 23. P. 2530-2532.

[12] Y. Otani, H. Miyajima, S. Chikazumi. IEEE Trans. Magn. (1989). V. 25. P. 3431-3433.

[13] The Science of Hysteresis / Eds G. Bertotti, I. Mayergoyz. Elsevier, Amsterdam (2006). V. II. P. 181-267.

[14] E.N. Kablov, O.G. Ospennikova, D.E. Kablov, V.P. Piskorskii, E.I. Kunitsyna, A.I. Dmitriev, R.A. Valeev, D.V. Korolev, I.I. Rezchikova, A.D. Talantsev, R.B. Morgunov. J. Appl. Phys. 117, 243903 (2015).

[15] E. Kablov, O. Ospennikova, V. Piskorskii, D. Korolev, A. Dmitriev, E. Kunitsyna, R. Morgunov. Eur. Phys. J. Plus 131, 40 (2016).

[16] R.B. Morgunov, E.I. Kunitsyna, V.V. Kucheryaev, V.P. Piskorskii, O.G. Ospennikova, E.N. Kablov. Eur. Phys. J. Plus 131, 344 (2016).

[17] А.И. Дмитриев, А.Д. Таланцев, Е.И. Куницына, Р.Б. Моргунов, В.П. Пискорский, О.Г. Оспенникова, Е.Н. Каблов. ЖЭТФ 150, 350 (2016).

[18] S.J. Lloyd, J.C. Loudon, P.A. Midley. J. Microscopy 207, 118 (2002).

[19] X.C. Kou, H. Kronmuller, D. Givord, M.F. Rossignol. Phys. Rev. B 50, 3849 (1994).

[20] H. Kronmuller, K.D. Durst, M. Sagawa. J. Magn. Magn. Mater. 74, 291 (1988).

[21] S.J. Newnham, J.P. Jakubovics, A.C. Daykin. J. Magn. Magn. Mater., 157/158, 39(1996).

[22] В.М. Рудяк. УФН 101, 429(1970).

[23] G.M. Jenkins, D.G. Watts. Spectral analysis and its applications. Holden-Day (1968). 525 p.

[24] N.J. Kasdin. Proc. IEEE 83, 802 (1995). 